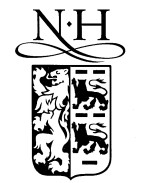

ELSEVIER

\title{
Dynamic Y-branched structures in quadratic nonlinear media
}

\author{
Maria C. Santos* \\ Universitat Politècnica de Catalunya, Department of Signal Theory and Communications, Lab of Photonics, Jordi Girona 1 y 3 , \\ 08034 Barcelona, Spain
}

Received 15 December 1999; received in revised form 13 March 2000; accepted 6 April 2000

\begin{abstract}
A selfsplitting of the beams entering a waveguide made of a quadratic nonlinear material is observed numerically. Relative $\pi$ phase differences between harmonics as positively contributing to linear diffraction are shown to trigger the effect. Formation of solitons out of this energy so that a Y-branched structure is nonlinearly induced, is shown to be dependent on input and material parameters. Simple setups should provide the means for experimental observation of the predicted phenomena. (C) 2000 Elsevier Science B.V. All rights reserved.
\end{abstract}

Keywords: Spatial solitons; Quadratic solitons; Beam splitting; Soliton switching; All-optical switching

As self-guided entities, spatial solitons provide a unique means for the control of light by light [1] which is central to all-optical technologies bound to expand network capacity and to revolutionize other fields of applications. Therefore, the interest arisen and the efforts dedicated to investigate their potential and properties. Light controlled soliton beam steering and splitting, spiralling, fusing and colliding has been predicted and in some cases observed, in a variety of setups and geometries [2], thus paving the way to soliton-based all-optical operation. Here we report numerical evidence of a new soliton-switching behavior, namely selfsplitting of input beams into several solitons in slab waveguides built up into quadratic nonlinear media.

\footnotetext{
*Fax: +34-93-4017232; e-mail: santos@tsc.upc.es
}

Since recalling old studies that predicted their existence [3], preliminar works demonstrated their scientific relevance [4,5], and the first experimental results were obtained [6,7], quadratic solitons have been a continuous source of new and interesting phenomena [8]. Considerable efforts have been devoted to shed some light into the fascinating nature of these nonlinear entities, but still they seem to keep a potential to be explored. In particular, as evidenced by the numerical simulations here presented, under certain circumstances, beam break-up may occur in the quadratic nonlinear crystal giving place to nonlinearly induced Y-branched structures similar to those due to Kerr-like nonlinearities $[9,10]$. In the quadratic case, larger nonlinear material responses allow to use longer pulse lengths thus avoiding undesirable effects related to two-photon absorption or group velocity dispersion, and reducing the complexity of the experimental setup required. 
The phenomenon of beam splitting in quadratic nonlinear media has already been discussed by Torres and Torner in the context of the NLSE approach (i.e. large wavevector mismatches) and in the presence of a certain Poynting vector walkoff, which hence acts as a perturbation to the NLSE causing the breakup of high power beams into lower order solitons [11]. The case that we study here has a different nature and it is observed numerically in other regimes of wavevector mismatch values, including negative ones, and with zero walkoff. The basis is to provide the system with input conditions that do not fit those of a nonlinear eigenmode so that initially a great imbalance is imposed between nonlinear phases rearrangement and linear diffraction. Such an imbalance determines the fields evolution towards an oscillating state with alternating diffraction and focusing periods. The quadratic system does not support these oscillating states and, as it is shown below, under some conditions the initial beam can give rise to two spatial solitons with opposite transverse velocities.

Leo and Assanto [12] have already studied this beam break up in type II configurations. Here different aspects of the selfsplitting mechanism are investigated with the aid of numerical methods. Since we aim at understanding the basics of the effect, monochromatic beam propagation in type I planar waveguide structures is considered. The reduced normalized equations that govern such a process are as follows,

$$
\begin{aligned}
& \mathrm{i} \frac{\partial a_{1}}{\partial \xi}-\frac{r}{2} \frac{\partial^{2} a_{1}}{\partial s^{2}}+a_{1}^{*} a_{2} \exp (-\mathrm{i} \beta \xi)=0, \\
& \mathrm{i} \frac{\partial a_{2}}{\partial \xi}-\frac{\alpha}{2} \frac{\partial^{2} a_{2}}{\partial s^{2}}-\mathrm{i} \delta \frac{\partial a_{2}}{\partial s}+a_{1}^{2} \exp (\mathrm{i} \beta \xi)=0,
\end{aligned}
$$

where $a_{1}$ and $a_{2}$ are the normalized amplitudes of the fundamental and second-harmonic waves, $r=$ -1 , and $\alpha=-k_{1} / k_{2}$. Here $k_{1,2}$ are the linear wavenumbers at both frequencies. In all cases $\alpha \simeq$ -0.5 , so we set $\alpha=-0.5$. The parameter $\beta$ is given by $\beta=k_{1} \eta^{2} \Delta k$ where $\Delta k=2 k_{1}-k_{2}$, is the wavevector mismatch, and $\eta$ is a characteristic beam width. The parameter $\delta$ accounts for the Poynting vector beam walk-off that occurs in birefringent media when light propagation is not along the crystal optical axes, and is given by $\delta=k_{1} \eta \rho, \rho$ being the walk-off angle. Bearing in mind non-critical and typical quasi-phase-matching settings in which Poynting vector walk-off is absent, we set $\delta=0$ in our numerical experiments. Finally, in Eqs. (1) the transverse coordinate is given in units of $\eta$, and the scaled propagation coordinate is $\xi=z / k_{1} \eta^{2}$.

From a qualitative viewpoint, the mechanism leading to the splitting may be understood as follows. First consider that for bright bell-shaped initial field profiles, in a wide variety of input and material conditions, during the first steps of the evolution, diffraction is compensated through the action of nonlinear parametric interaction so that energy remains mainly concentrated in the center of the profiles and a bright bell-shaped soliton is formed [1315]. Some situations can be found however, in which initially the effect of the nonlinear interaction adds up to that of diffraction so that an energy flow is set up going from the center towards the wings of the profile. Under suitable conditions, the local energy gain experienced at each side of the profiles may allow to reach an equilibrium such that energy is focused towards off-center transverse points, providing steadiness to the initial center-sides transverse energy flow and determining the splitting of the input beam.

As noted by Leo et al. [12], the phase profiles play a key role in the splitting mechanism. To make it evident, Eqs. (1) may be understood in terms of local amplitudes and phases evolution. By writing $a_{i}=U_{i} \exp \left(\psi_{i}\right)$, one obtains

$$
\begin{aligned}
& \frac{\partial\left(U_{1}^{2}\right)}{\partial \xi}=r\left(\psi_{1_{s}} U_{1}^{2}\right)_{s}+2 U_{1}^{2} U_{2} \sin (\Delta \psi), \\
& \frac{\partial\left(U_{2}^{2}\right)}{\partial \xi}=\left(\left(\alpha \psi_{2_{s}}+\delta\right) U_{2}^{2}\right)_{s}-2 U_{1}^{2} U_{2} \sin (\Delta \psi),
\end{aligned}
$$

with $\Delta \psi$ the relative phase difference verifying $\Delta \psi$ $=2 \psi_{1}-\psi_{2}+\beta \xi$ and subindices denoting the derivatives. Physically, the last terms in Eqs. (2) account for interharmonics energy exchange while first ones keep track of the amount of local energy that is redistributed in the transverse direction within 
the same harmonic. For the stationary bell-shaped solutions known to date, in the absence of Poynting vector walk-off both terms in each expression must vanish with $\Delta \psi=0$ and with plane wavefronts, while in the presence of it, they cancel each other featuring non-trivial transverse phase modulations [16].

For the evolution of the local phases one has $\psi_{1_{\xi}}=-\frac{r}{2} \frac{U_{1_{s s}}}{U_{1}}+\frac{r}{2}\left(\psi_{1_{s}}\right)^{2}+U_{2} \cos (\Delta \psi)$,

$$
\begin{aligned}
\psi_{2_{\xi}}= & -\frac{\alpha}{2} \frac{U_{2_{s s}}}{U_{2}}+\frac{\alpha}{2}\left(\psi_{2_{s}}\right)^{2}+\delta \psi_{2 s} \\
& +\frac{U_{1}^{2}}{U_{2}} \cos (\Delta \psi) .
\end{aligned}
$$

For the zero walk-off configurations that we consider here, essentially, the contribution of the nonlinear interaction is gathered in the lasts terms in these expressions while first and second terms account
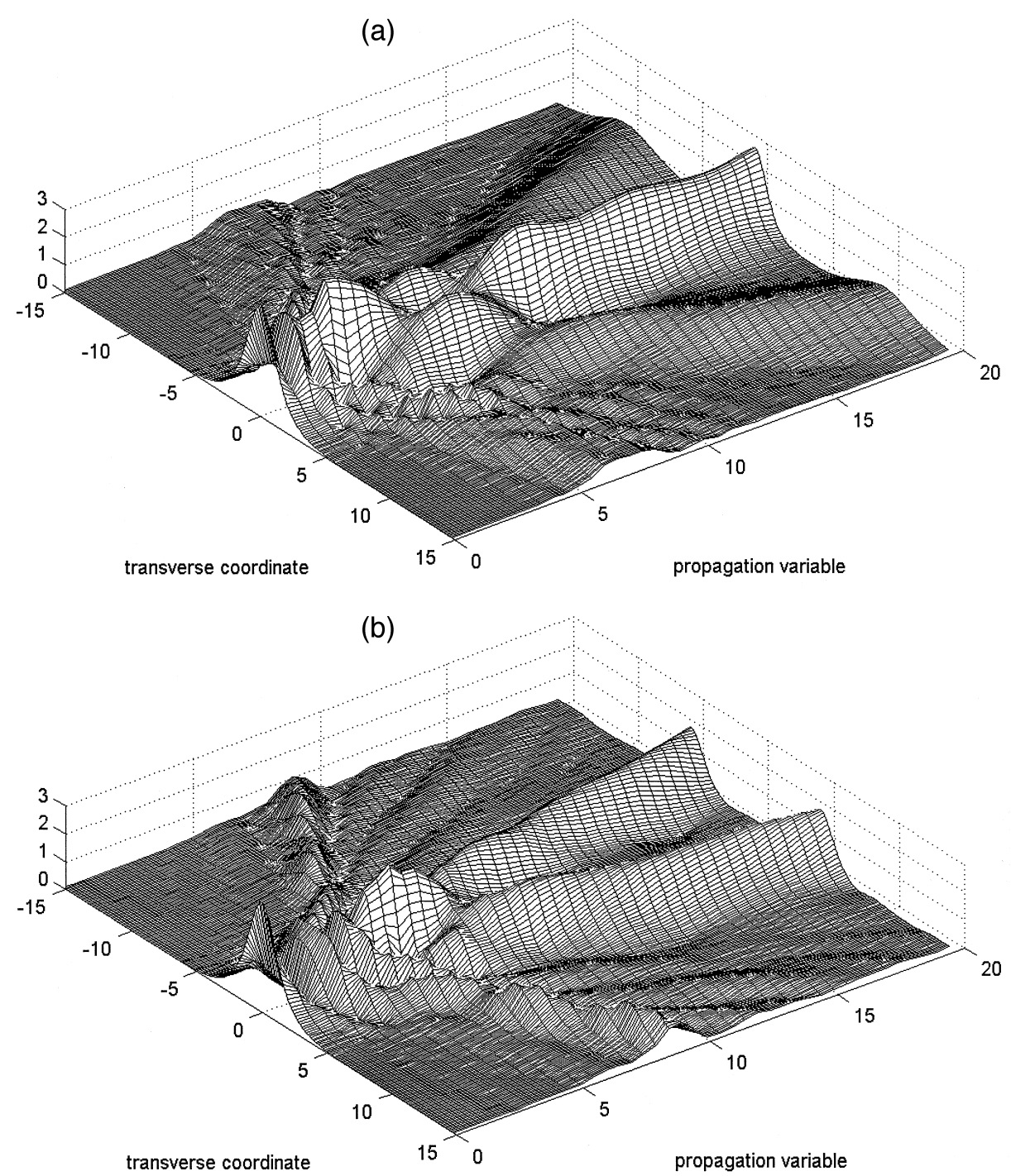

Fig. 1. Amplitude fundamental field profiles evolution when imposing a $\pi$ input difference between harmonics. In both cases inputs are as in (4) with $A=4, B=1, \phi_{0}=\pi$. (a): $\beta=4$, (b): $\beta=2$. 

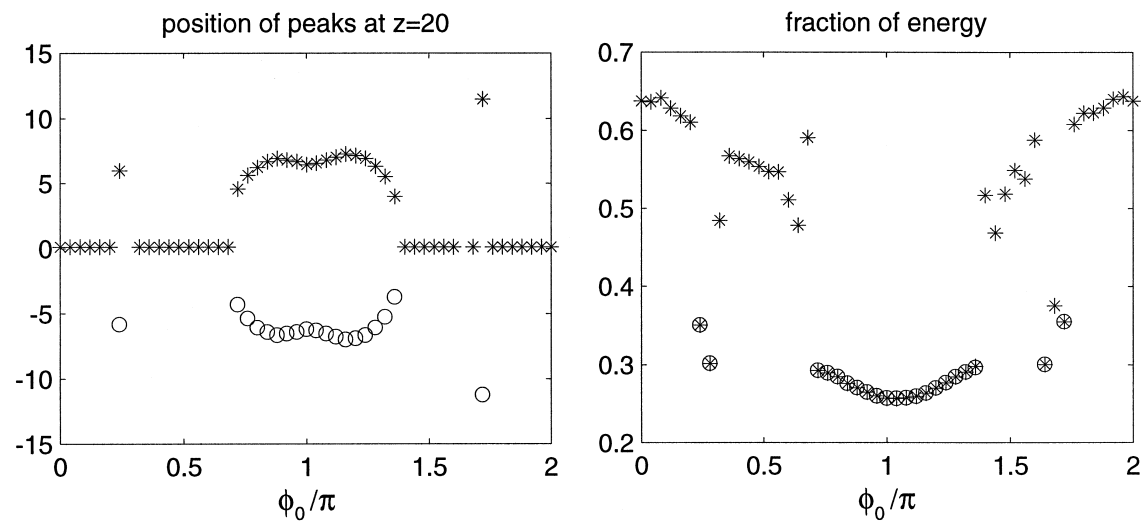

Fig. 2. Position of peaks found at the output face of a 20 normalized units length waveguide (left) and fraction of energy alloted to each one as a function of the initial phase difference imposed between the two harmonics for $\beta=-0.5$, inputs are as in (4) with $A=5, B=0.5$. Each symbol identifies a point of maximum field intensity so that equal symbols make reference to the same peak.

respectively for linear diffraction and transverse displacements of the beam. Relevant to gain some insight into the splitting mechanism is the fact, exposed through the local energies and phases forms of the governing equations i.e. (2) and (3), that an essentially differentiated behavior is expected depending on the sign of the parametric interaction term. Following (3), when the sign of the interaction is positive, a local increase of the phase constants in more energetic transverse points determines a phase gradient which as denoted by the first terms in (2), drags energy towards these points enhancing the focusing of the initial bright beam. Conversely when this sign is negative, more energetic points experience a decrease of their phase constants that causes there a defocusing.

The numerical simulations using input beams with the generic form

$a_{1}(\xi=0, s)=A \operatorname{Sech}(s)$,

$a_{2}(\xi=0, s)=B \operatorname{Sech}(s) \exp \left(\mathrm{j} \phi_{o}\right)$,

indeed confirm that there is a link between the splitting of the input beam and near- $\pi$ relative phase difference values. Fig. 1 shows an example of such a behavior, obtained with $\phi_{o}=\pi$. In case (a) the energy initially dragged towards the wings is too low for reaching there an equilibrium, and thus focusing back towards the center prevents beam break-up so that a single soliton output is obtained. A lower $\beta$ value in case (b) makes it easier to lock the phases up in the profile wings so that $\Delta \psi=0$ and two solitons are found at the output waveguide face.

To investigate the potential of such an splitting for all-optical switching we carried out a series of numerical experiments considering different values of the various parameters involved and computed the number and position of peaks and a measure of the fraction of the total input energy that is allotted to each one after a normalized propagation distance of $\xi=20$ equivalent to 40 diffraction lengths, defined as $L_{\mathrm{d}}=k_{1} \eta^{2} / 2$. With eyes to experimental observa-

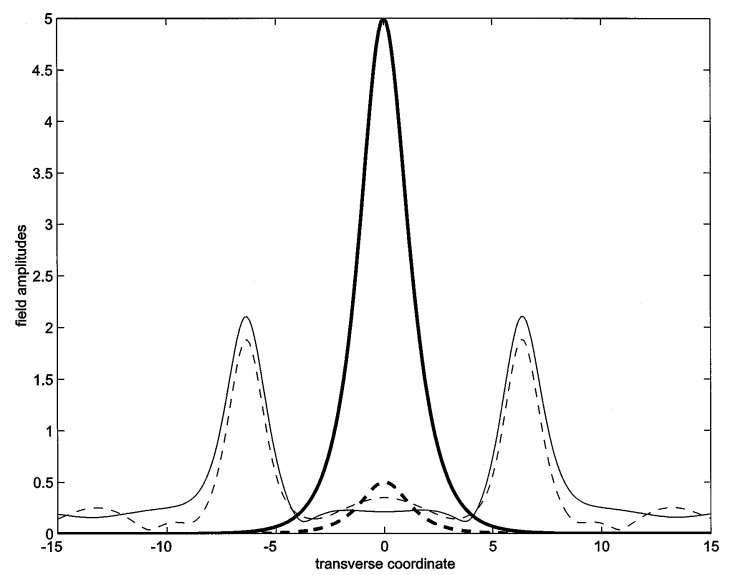

Fig. 3. Input (thick lines)-output (thin lines) transverse field profiles characteristic in the 20 normalized units long waveguide for inputs as in (4) with $\beta=-0.5, A=5, B=0.5, \phi_{0}=\pi$. Continous lines represent fundamental field profiles while dotted lines correspond to the second harmonic. 

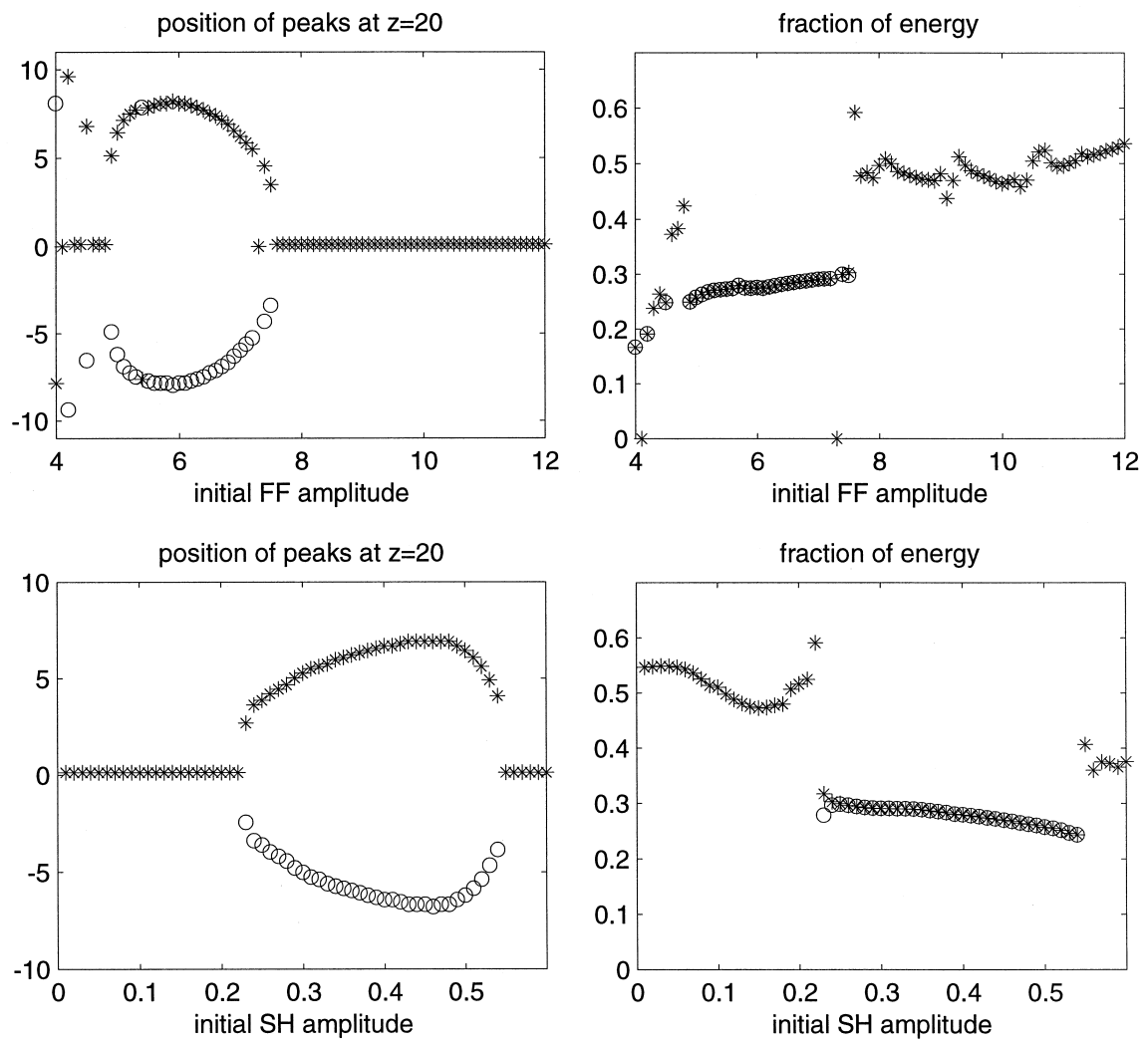

Fig. 4. Same as in Fig. 2 with fixed input phase difference $\phi_{0}=\pi$, showing the input amplitudes dependence of the splitting. Top: $B=0.5$, bottom: $A=5.5$.

tion of the phenomena, the simulations consider a Ti:indiffused slab waveguide in PPLN fabricated as described in [17] with inputs from an Nd:Yag laser source, featuring working wavelengths of $\lambda \sim 1 \mu \mathrm{m}$, focused to $\eta \sim 15 \mu \mathrm{m}$ beam widths. Corresponding diffraction lengths are on the $1.3 \mathrm{~mm}$. range resulting in required waveguide lengths of about $5 \mathrm{~cm}$. well within the reach of today's existing technology. In
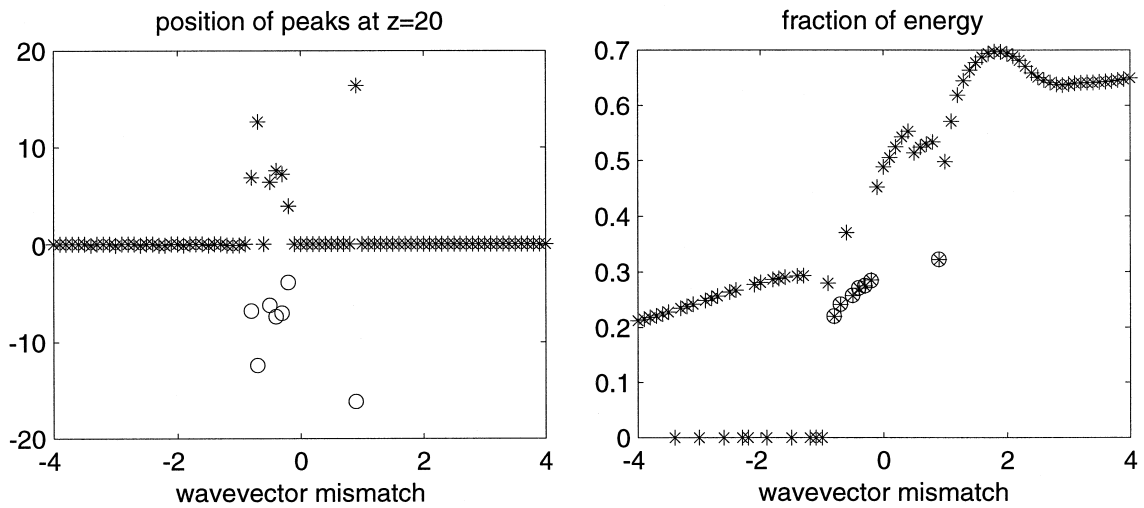

Fig. 5. Same as in Fig. 4 with $\phi_{0}=\pi$ showing the wavevector mismatch dependence of the splitting. 
such an experimental setup, input power levels up to 20 normalized units as used in the numerical experiments, require peak fields on the order of 100 $\mathrm{KV} / \mathrm{mm}$, meaning peak intensities in the waveguide of some few $\mathrm{GW} / \mathrm{cm}^{2}$. The use of a KDP crystal before a variable-pressure nitrogen cell for input relative phase control as in [7] is envisioned for the experiments which require a second harmonic input.

In a first set of numerical experiments we considered the input phase difference, $\phi_{o}$, to be the parameter of control of the dynamic Y-branched structure. Having in mind configurations of practical interest, the input intensity of the second harmonic was set to be on the range of the $1 \%$ with respect to that of the fundamental. Fig. 2 shows some of the results. A broad splitting margin around $\pi$ input phase differences is obtained with reasonable energy efficiencies in terms of input energy. A look at the input-output field profiles characteristic in Fig. 3 reveals neat soliton formation at each side of the center.

Another case of interest is that in which the splitting is controlled through the energy contained in one of the input beams. The characteristics displayed in Fig. 4 as featuring two distinct regimes for splitting and single-output behavior with moderate energy efficiencies, show the potential of the splitting effect here studied for switching applications with power control.

The mutual influence between amplitudes and phases made evident by expressions (2), (3) help to understand some of the features displayed by the beam splitting, i.e. the fact that numerically it is observed for a certain range of input energies and amplitude relations. It can be argued that an enough powered input beam or with a certain amplitudes relation may allow the necessary phases rearrange-
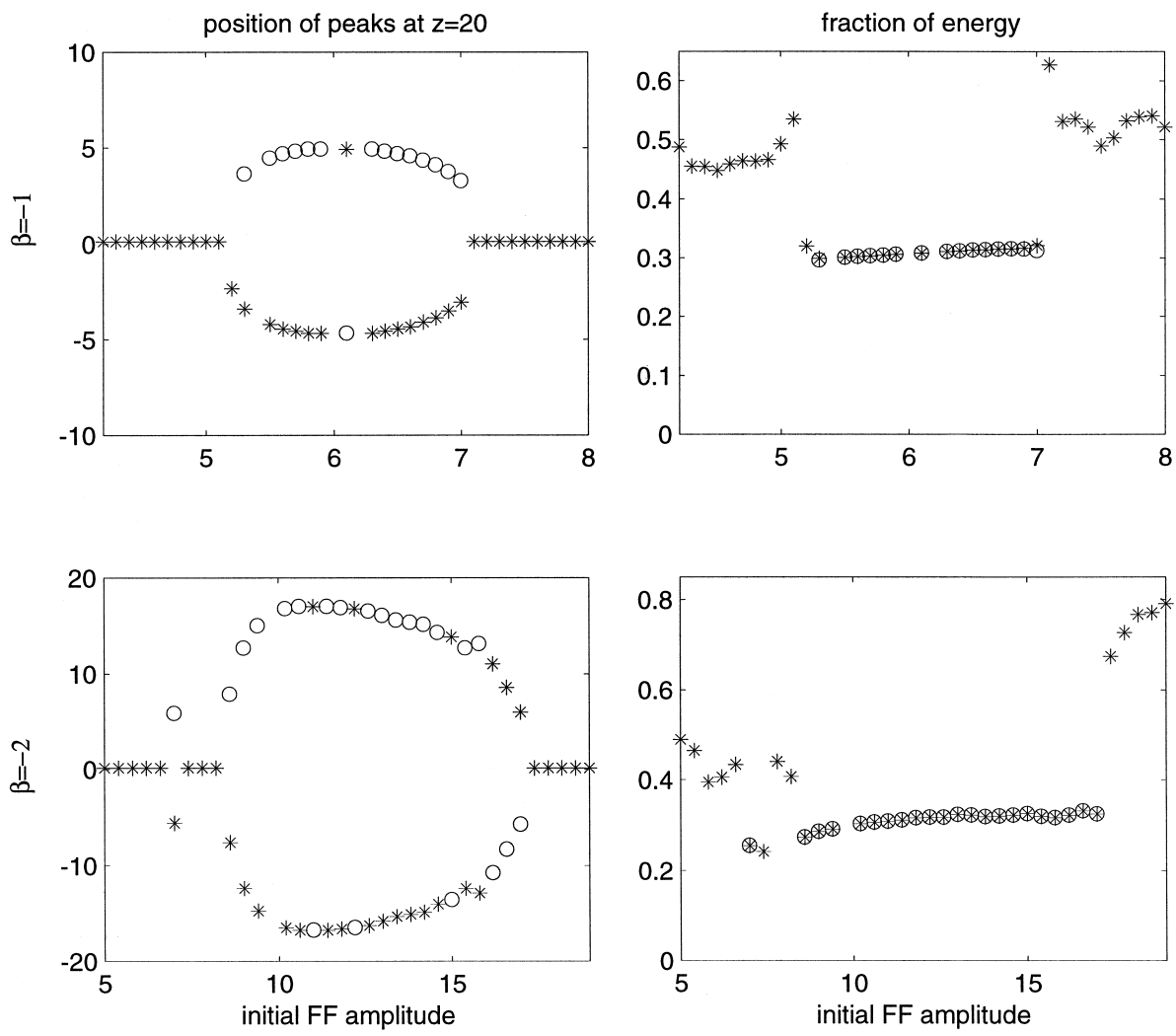

Fig. 6. Position of peaks found at the output face of the crystal waveguide (left) and fraction of energy alloted to each one as a function of input fundamental amplitude for two different values of the wavevector mismatch. Top: $\beta=-1$, bottom: $\beta=-2$. Inputs as in (4) with $\phi_{0}=0$ and $B=0$. Equal symbols identify the same field maximum. 
ment required to compensate the initial imbalance so that only one soliton is formed. In contrast, a low powered beam either is not capable of endowing the system with enough diffraction-focusing mismatch to drive it out from the one soliton state or else, it is not capable of forming two solitons, and the energy disperses into radiation. Of course the energy levels and amplitude relations required for any state strongly vary depending on the wavevector mismatch. This wavevector mismatch dependence is critical in this case as shown in Fig. 5. Therefore a mechanism for controlling the wavevector mismatch value should be provided if the phenomenon is to be of any applicability to switching. Conversely, other applications may take advantage of this critical dependence as an experimental indicative of the wavevector mismatch value effective in the sample. For correct interpretation of the results in Fig. 5 we note that zero output energy values indicate no neat soliton formation at the end face of the waveguide.

When looking for other cases of beam splitting, extensive numerical experiments made evident that
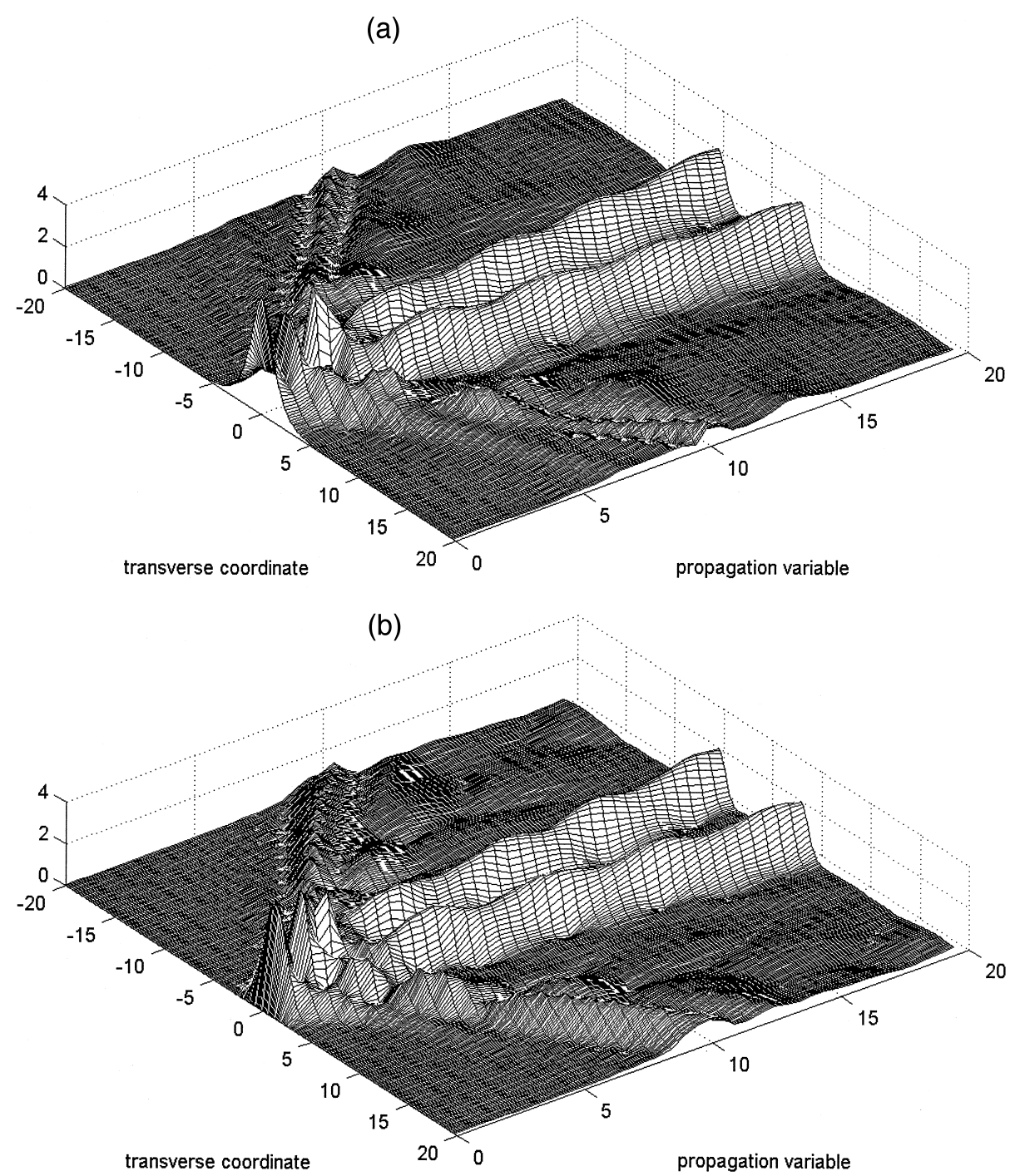

Fig. 7. Fields evolution for an input as in (4) with $A=5.5, B=0, \phi_{0}=0$ and $\beta=-1$. Top: fundamental field, bottom: second harmonic. 


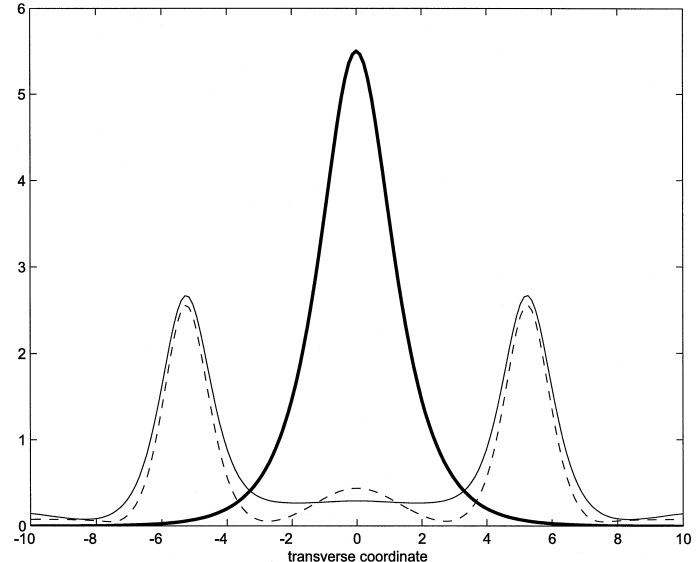

Fig. 8. Input field profiles (thick lines) and output field profiles after $\xi=20$ normalized propagation distances (thin lines) for an input as in (4) with $\phi_{0}=0, A=5.5, B=0, \beta=-1$. Continous lines represent the fundamental field while dotted ones correspond to the second harmonic. for beam break-up to take place not only could the relative $\pi$ phase difference, which as nonlinearly enhancing the dispersion of energy from the center was the origin of the beam splitting, be imposed initially, but it also could be reached through the dynamics with bright input in-phase harmonics with amplitudes in complete mismatch from those allowing formation of bright solitons traveling in the straight direction. A qualitative explanation of this characteristic stems from expressions (2), (3) taking into consideration that in that case, local interharmonics energy exchanges tend to rearrange the amplitudes such that they drive the system towards the stationary (no energy exchange) condition with $\Delta \psi$ $=\pi$, instead of $\Delta \psi=0$, and with more energetic points having faster interharmonics energy exchange rates, and therefore reaching faster to this point. According to (3), $\Delta \psi=\pi$ determines a tendency to nonlinearly cause a decrease of the local phase values thus setting a transverse phase gradient up that
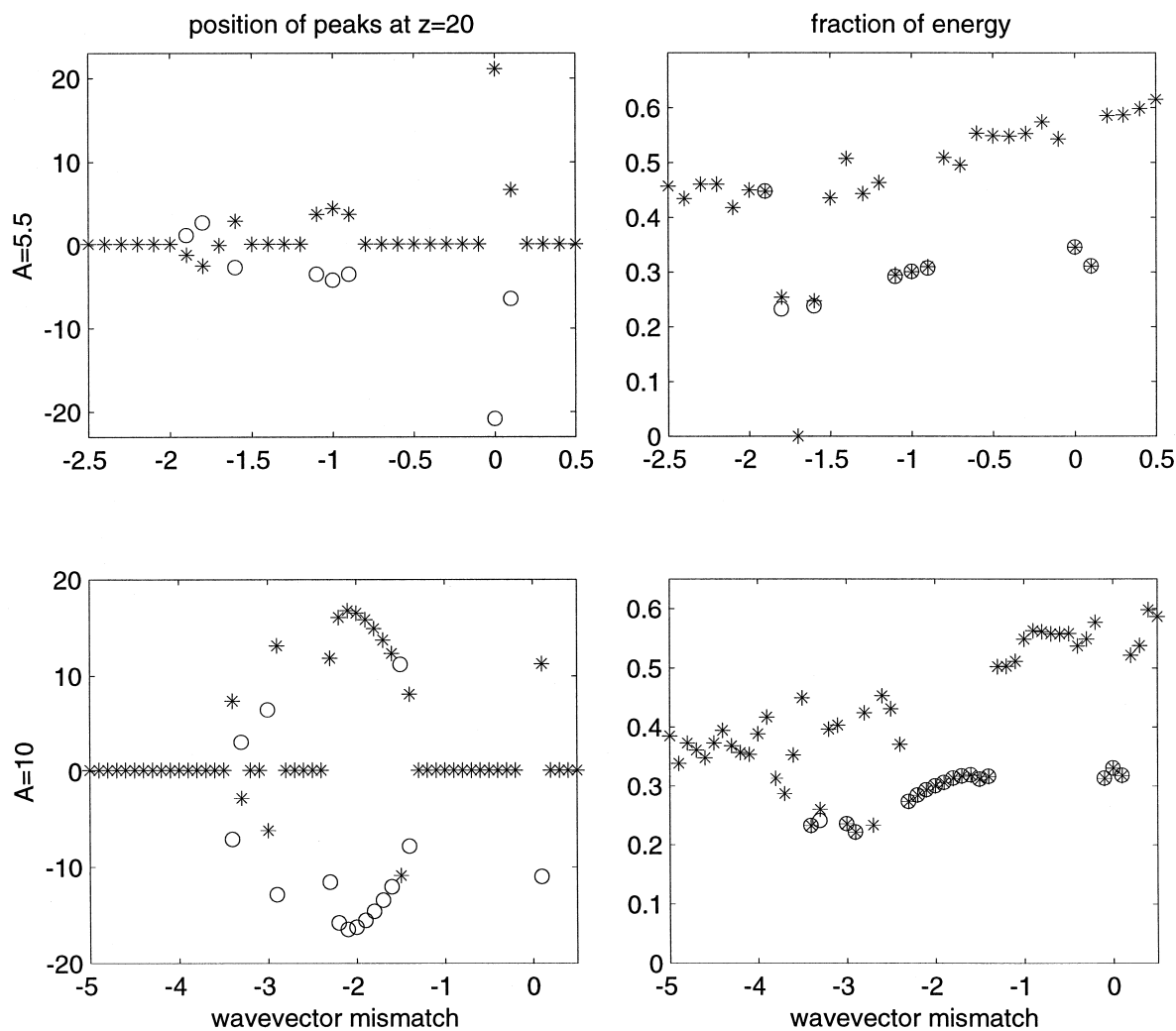

Fig. 9. Wavevector mismatch dependence of the splitting. Inputs as in (4) with $B=0$ and $\phi_{0}=0$. (a) $A=5.5$ (b) $A=10$. 

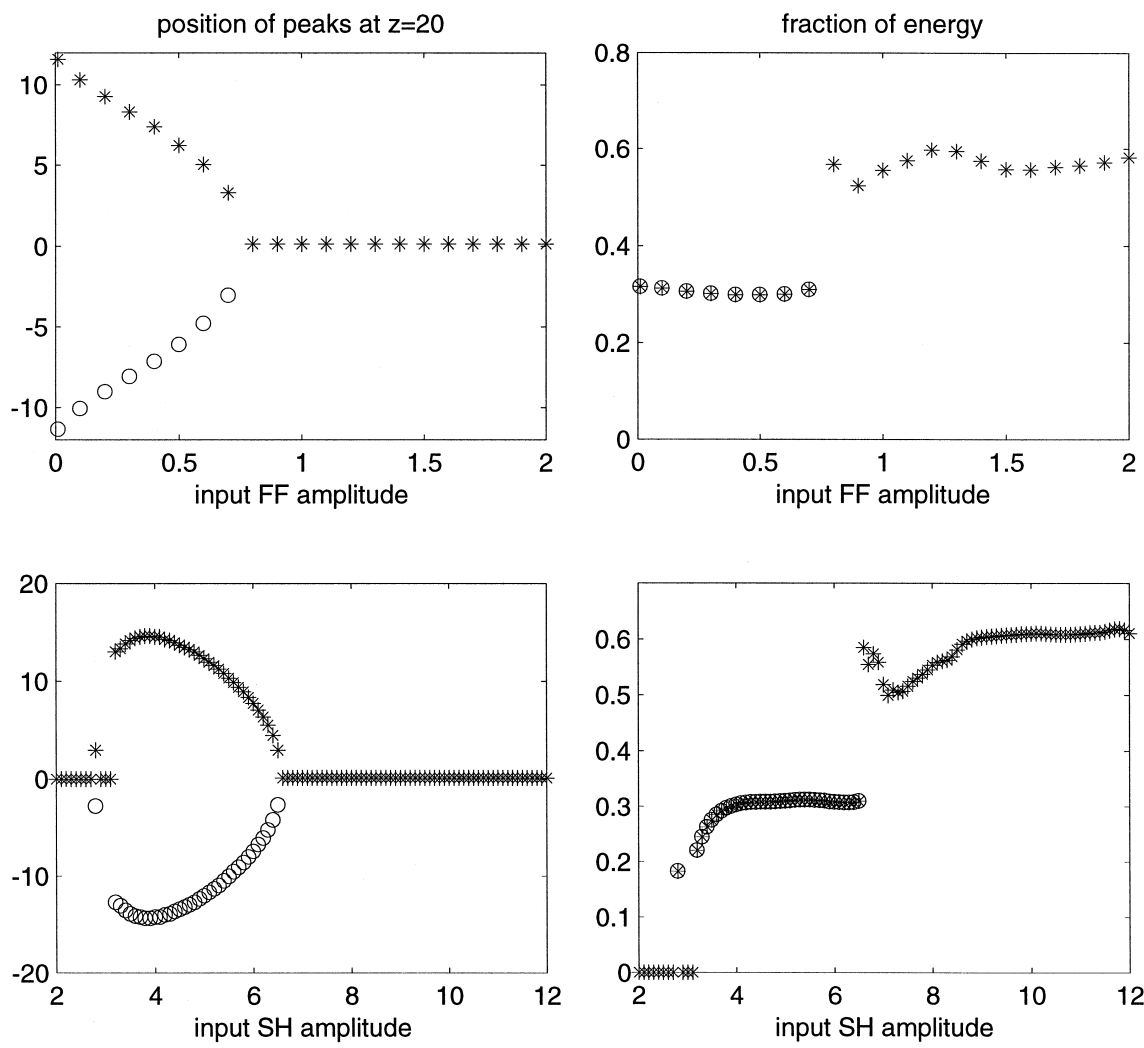

Fig. 10. Position of peaks found at the output face of the crystal waveguide (left) and (right) fraction of energy alloted to each one as a function of input fundamental amplitude (top, $B=5.5$ ) and as a function of input second harmonic amplitude (bottom, $A=0.1$ ). In both cases $\beta=3$ and inputs as in (4) with $\phi_{0}=0$.

tends to drag energy out of from more energetic transverse points towards less energetic ones, i.e., for the bright input profiles used in this work, from center towards off-center points. Then, either the energy exchange at the center is fast enough to recover the proper amplitude relation to get $\Delta \psi=0$
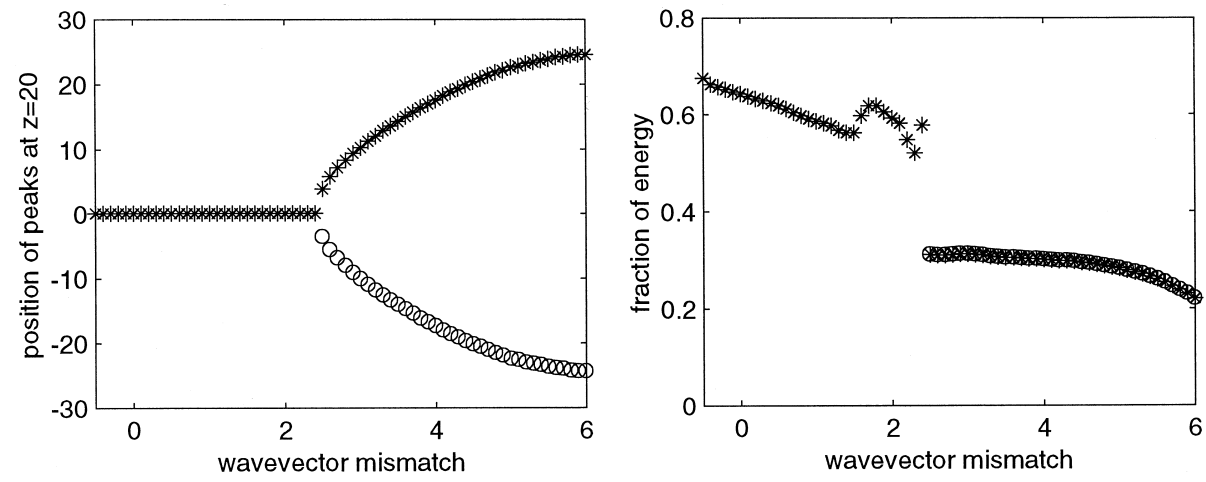

Fig. 11. Wavevector mismatch dependence of the splitting effect. Left: position of peaks found at the output face of the 40 diffractions lengths long waveguide and right: fraction of input energy alloted to it. Inputs as in (4) with $\phi_{0}=0, A=0.1, B=5.5$. 
and thus the increase in the local phase values required to have a significant amount of the energy back so that a single bright soliton is formed (Fig. 1a); or else it may be off-center transverse points, progressively speeding their interharmonic energy exchange up owing to the injection of energy coming from the initial off-center energy dragging, to reach faster to the $\Delta \psi=0$ point causing the splitting of the input beam, (Fig. 1b).

According to the above, if only the fundamental is used as input, negative phase mismatches need to be used if any beam splitting is to be observed. The results in Fig. 6 are an example. A moderately large input amplitudes margin is obtained which leads to beam splitting with good energy efficiencies thus revealing the potential of the process for power dependent switching operation with a single beam input. As seen, increasing the phase mismatch causes a significant broadening of the splitting interval which shifts towards higher amplitude values while giving rise to larger angles of beam propagation with respect to the crystal axis. Fig. 7 gives a detail of the beam evolution which evidences the strong reshaping taking place in the first steps of propagation while Fig. 8 illustrates the input-output field profiles characteristic.

To further explore this phase mismatch dependence of the splitting and elucidate how sensitive it is to small changes in its value, we plotted the results against phase mismatch variation. The results in Fig. 9 reveal a quite strong sensibility with changes on the 0.1 order determining the evolution from single to multi-peaked outputs. Broader margins (on the 0.7 order) can be obtained with larger input amplitudes as seen in case (b).

In a third set of experiments the down-conversion case was considered. With positive phase mismatches we checked out that formation of Y-branched
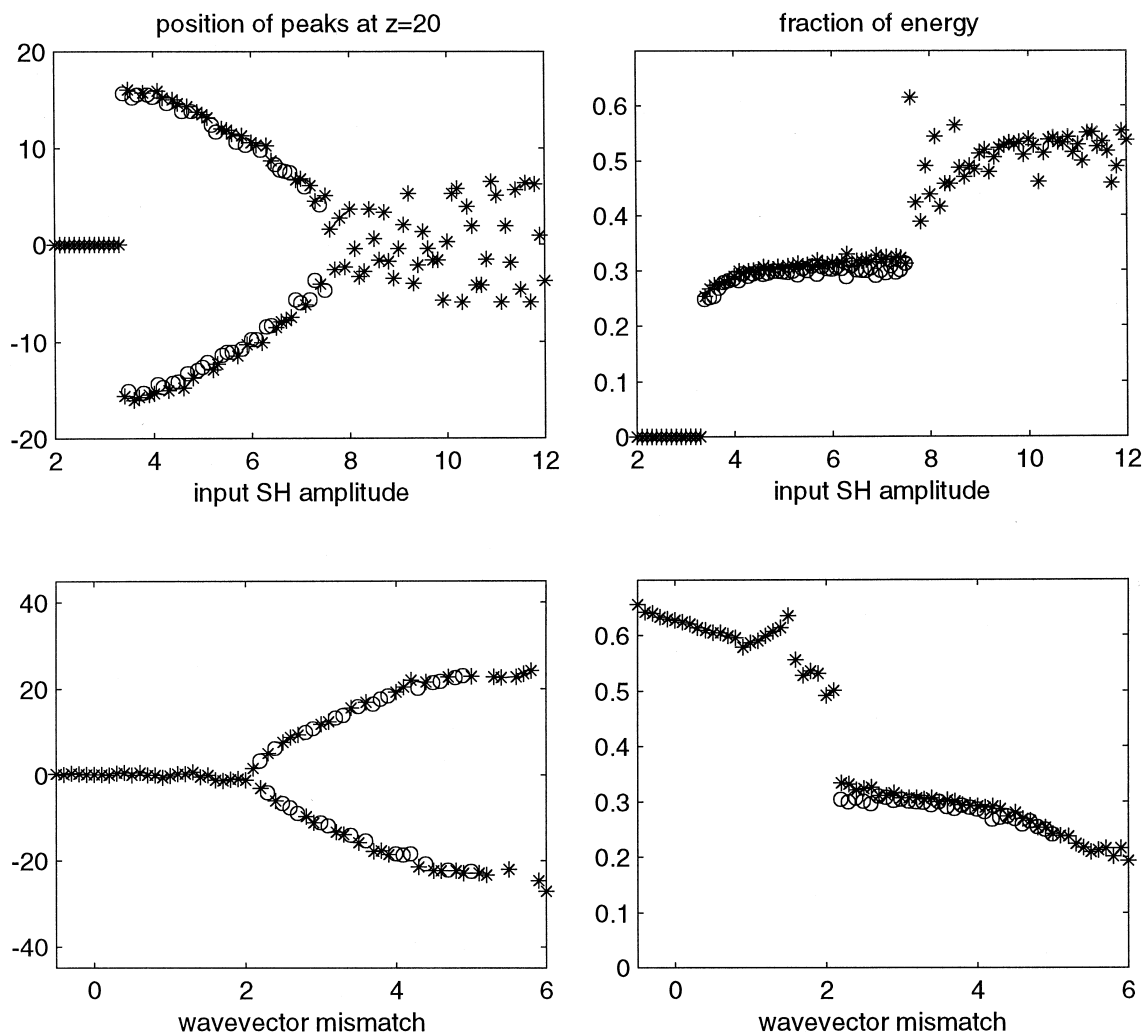

Fig. 12. Same as in Fig. 10 bottom, and Fig. 11 but considering a gaussian noise of 0.001 normalized power as input fundamental seed. 
structures may be controlled through the power contained either in the second harmonic beam or in a low power fundamental. Examples are shown in Fig. 10. Not only the splitting effect has proved robust against phase mismatch variations in this case, as evidenced by the results in Fig. 11, but it also displays a quite nice characteristic for all-optical switching operation with two differenciated and quite broad regimes of operation featuring respectively single and double beam outputs.

In order to mimic realistic experimental conditions and verify the usefulness of the effect in practical setups, very low power gaussian noisy inputs at the fundamental frequency were used to seed the conversion process. The outcome shown in Fig. 12 confirms preservation of the basic characteristics obtained with deterministic inputs.

In conclusion, we have observed numerically the formation of soliton induced Y-branched structures in slab waveguides made of quadratic nonlinear media and verified its dependences against input and material conditions while identifying possibilities for use in all-optical switching devices design. We have confirmed that efficient control of beam break-up may be performed either through the input phase difference between harmonics or by the power contained in either beam. From the three different schemes considered for beam splitting control, namely input phase differences, up-conversion and down-conversion, only the latter has proven to be robust against phase mismatch variations. Experimental observation of the effects here described should be possible in quite simple setups by careful adjustment of the experimental conditions, thus opening the door to new ideas for the design of all-optical switching devices.

\section{Acknowledgements}

The author gratefully acknowledges numerous valuable remarks by Lluis Torner and his encouragement of the work which has been supported by the Spanish Government under grant PB95-0768.

\section{References}

[1] A. Snyder, F. Ladoceur, Optics and Photonics News (Feb. 99) 35.

[2] M. Segev, G. Stegeman, Physics Today 51 (Aug. 1998) 42.

[3] Yu.N. Karamzin, A.P. Sukhorukov, Sov. Phys. JETP 41 (1976) 414.

[4] C.R. Menyuk, R. Schiek, L. Torner, J. Opt. Soc. Am. B 1 (1994) 2434.

[5] L. Torner, Opt. Commun. 114 (1995) 136.

[6] R. Schieck, Y. Baek, G.I. Stegeman, Phys. Rev. E 53 (1996) 1138.

[7] W.E. Torruellas, Z. Wang, D.J. Hagan, E.W. Vanstryland, G.I. Stegeman, L. Torner, C.R. Menyuk, Phys. Rev. Lett. 74 (1995) 5036.

[8] G.I. Stegeman, D.J. Hagan, L. Torner, Opt. Quantum Electron. 28 (1996) 1691.

[9] D. Burak, R. Binder, J. Opt. Soc. Am. B 1 (1997) 1458.

[10] A.W. Snyder, A.V. Buryak, D.J. Mitchell, Opt. Lett. 23 (1998) 4.

[11] J.P. Torres, L. Torner, Opt. Quantum Electron. 29 (1997) 757.

[12] G. Leo, G. Assanto, Opt. Commun. 146 (1998) 356.

[13] L. Torner, C.R. Menyuk, G. Stegeman, Opt. Lett. 19 (1995) 1615.

[14] L. Torner, C.R. Menyuk, G.I. Stegeman, J. Opt. Soc. Am. B 1 (1995) 889.

[15] L. Torner, E.M. Wright, J. Opt. Soc. Am. B 1 (1996) 864.

[16] L. Torner, D. Mihalache, D. Mazilu, M.C. Santos, N.N. Akhmediev, J. Opt. Soc. Am. B 1 (1998) 1476.

[17] J. Amin, V. Pruneri, J. Webjorn, P.St.J. Russell, D.C. Hanna, J.C. Wilkinson, Opt. Commun. 135 (1997) 41. 